\title{
Importance Of Pilot Study And Data Quality Control In Designing Complex Training Programs For Young Athletes
}

\author{
Rojapon Buranarugsa ${ }^{*}, 1,3$, José Oliveira ${ }^{2}$ and José Maia ${ }^{1}$ \\ ${ }^{I}$ Center of Research, Education, Innovation and Intervention in Sport (CIFI2D), Faculty of Sport, University of Porto, \\ Porto, Portugal \\ ${ }^{2}$ Research Center in Physical Activity, Health and Leisure (CIAFEL), Faculty of Sport, University of Porto, Porto, Portugal \\ ${ }^{3}$ Khon Kaen University, Khon Kaen, Thailand
}

\begin{abstract}
The aims of this pilot study were to use various muscular strength and performance evaluation procedures to assess the preliminary responses from young Thai male athletes to strength training programs, and to apply data quality control to assess data reliability. Sixteen young Thai male athletes aged 13-17 years from the Khon Kaen Sport School were sampled: eight were soccer players and eight were track and field athletes. Subjects were divided into two groups: a complex training group $(n=8)$ and a resistance training group $(n=8)$. The pilot intervention program was applied twice a week during two weeks. Maximum strength, anaerobic power, vertical jumps, 40-yard sprints, agility and sports skills were measured before and after the two-week training programs. As expected no significant changes were observed in all measured variables from pre- to post-testing in both groups ( $p>0.05)$; mean differences $(\Delta)$ between pre- and post-testing of all variables were close to zero, and high reliability values were observed. All subjects handled well the training programs and assessments. No injuries occurred during all training and testing sessions. The training programs and testing procedures were suitable and safe for young male Thai athletes. Results from statistical analysis showed the achievement of high quality data. Further research in this cohort can be done under a well-designed training program and close supervision by researchers.
\end{abstract}

Keywords: Complex training, data quality control, physical performance, resistance training, strength, young, athletes.

\section{INTRODUCTION}

Youth strength training is becoming popular as a suitable way, among others, to enhance performance in sports and reduce potential injury, and has been incorporated in many sports-oriented annual plans. Although musculoskeletal injury risk and growth disturbance from growth plate injury are major concerns in youth strength training, recent evidence has supported the idea that a strength-training program can be safe for young people if that program is properly designed and participants are closely supervised. A review study clearly indicated that resistance training has no negative influence on the growth and maturation of youngsters [1]. Moreover, there are now several position statements from important medical and scientific organizations favouring quality supervised strength training programs to enhance sports performance, prevent injury and improve the general health of children and youth involved in sports [2, 3].

Strength training programs for youth can be designed in several forms, the most effective and widely used in youth strength training researches are resistance and plyometric training [2]. Available data showed the benefits of resistance training programs when combined with sport-specific training in youth [4-6]. For example, significant strength and

*Address correspondence to this author at the Center of Research, Education, Innovation and Intervention in Sport (CIFI $\left.{ }^{2} \mathrm{D}\right)$, Faculty of Sport, University of Porto, Porto, Portugal; Tel: +351 964663997;

Fax:+351 225500689; E-mail: rojapon@hotmail.com physical performance improvements of adolescent soccer players were reported when combined with specific soccer and resistance training programs [4]. Plyometric training studies in youth reported changes in various types of performance such as in vertical jump, sprint, agility, and sprint cycling tests following training programs $[7,8]$. Recently, a position statement paper recommended that, when designing strength training programs for youth, coaches should use training loads in the range of $50-85 \%$ of $1 \mathrm{RM}$ for each exercise, using 1-3 sets $\times 6-15$ repetitions 2-3 days a week [2].

Another effective strength training modality, which is becoming popular is complex training. Complex training is best described as a strength and power training method that combines resistance and plyometric training in the same training session $[9,10]$. Available information demonstrates the positive effect of complex training on strength and physical performance in pre pubertal boys [11] and explosive strength and sprint performance when combined with routine training in young athletes $[12,13]$.

Although there is evidence supporting the idea that children and adolescents can obtain real benefits from participating in complex training programs, there are gaps in the literature, namely regarding the effects of complex training methods on strength in young athletes and including topics such as program design, physical performance assessment and responses to complex training programs from young athletes engaged in different types of sports. Furthermore, and contrary to what happens in USA and some Europeans 
countries, there is no research tradition in Thailand for investigating the effects of complex training or resistance training programs, or a combination of both, in young athletes. In addition, available research concerning the design, implementation, data quality control and interpretation of complex training in youth is scarce. Thus, before implementing any experimental strength training study, it would be of importance to provide suitable information regarding these issues. In addition there is not much information, if any, about methodological sound strength training programs evidence-based, nor a consistent tradition in data quality control prior to the implementation of such programs in young Thai athletes.

This report deals primarily with a pilot study centred on the designing of the strength training programs, data quality control and adequate statistical analysis. These primarily results will provide enough information about the validity of short to medium term intervention programs, and this information may be useful to coaches and athletes if they are highly interested in successful training methodologies aiming a high level performance. With these ideas in mind, a study was designed aiming to evaluate how young Thai male athletes respond to complex and resistance exercises in its pilot phase (two weeks), namely to examine the suitability of training programsand to assess data reliability when using diverse testing procedures. It can also provide a suitable template for future research using experimental training programs with young athletes. The linking hypothesis of this pilot study is that high reliable information can be achieved within highly designed (i.e., supervised and monitored) strength training programs. Furthermore, we wanted to subjectively assess from athletes and coaches the suitability of the programs and the testing procedures since this was the first time that such a training program took place.We are of the position that without systematic pilot studies about distinct short-duration training programs, persuasive data and equipment controls, no sound and relevant results will be provided to coaches and athletes when implementing such programs in their annual plans.

\section{METHODS}

\section{Subjects}

Subjects in this study were 16 young Thai male athletes aged 13-17 years from the Khon Kaen Sport School in Khon Kaen. Of the boys, eight were soccer players (SOC) from several soccer field positions (defender, midfielder, winger and striker) and eight were track and field athletes (TF)from several events (sprinter, hurdle, long jump, shot put and javelin throw. The subjects were further divided into two groups: a complex training group (CT: $\mathrm{n}=8 ; 14.4 \pm 1.5$ years) and a resistance training group (RT: $\mathrm{n}=8 ; 14.4 \pm 1.1$ years). The groups consisted of an equal number of both sport types (SOC; $\mathrm{n}=4, \mathrm{TF} ; \mathrm{n}=4$ ). Soccer players were trained by the same coach but track and field athletes were trained by different coaches according to their specialty. All subjects were involved in the training routine program for six days each week and participated in their regular school classes five days a week.

Prior to the study, all subjects and coaches were informed about the purposes of the study. Written informed consent was obtained from the parents. The study was conducted according to the declaration of Khon Kaen University, Khon
Kaen, Thailand and was approved by the Ethics Committee of the Faculty of Sport, University of Porto, Porto, Portugal.

\section{Research Procedure}

\section{Training Program}

As study was set to evaluate the feasibility of the complex and resistance training programs, no significant changes were expected in any physical performance variables. Thus, both complex and resistance training programs were designed to last only two weeks with two sessions per week. Training programs were part of normal daily training routines of all athletes, as follows:

1. A complex training program consisting of a combination of resistance training and plyometrics. Resistance and plyometric exercises as well as training intensity and volume used in training programs have been recommended for practicability, effective and safety with youth $[2,14,15]$. Throughout the study training intensity and volumes for resistance training were constant ( $70 \%$ of $1 \mathrm{RM} ; 2 \mathrm{x} 12)$ as well as for plyometric training ( $2 \times 10$ reps $)$. All subjects were evaluated for 1RM in all six resistance exercises before training in order to define target training load ( $70 \%$ of $1 \mathrm{RM})$. The order of the resistance exercises was as follows: bench press, arm curl, lat pull down, leg press, knee curl, and leg extension. Plyometric exercises, which were performed after the resistance exercises, consisted of medicine ball chest pass, medicine ball overhead throw, medicine ball backward throw, rim jump and tuck jump. This program is presented in Table $\mathbf{1}$.

2. A resistance training program consisting of only normal resistance exercises as used in the complex training program. Training intensity and volume were also equal to the complex training group. This program is presented in Table $\mathbf{1}$.

Both the complex and the resistance training programs were performed before the routine training programs of the school in the afternoon during a pilot program consisting of two sessions per week for two weeks on non-consecutive days. The duration of each training session was between 45 to 60 minutes.

\section{Testing Procedures}

All subjects performed all tests in one session during the morning for both pre and post-tests, ordering to the following sequence: 40-yard sprint, T-test, countermovement jump, squat jump, sport skills, 1RM bench press, 1RM leg press, and Wingate test. Subjects performed skill tests according to their sport specific type, passing and shooting skills for soccer player and medicine ball throw and standing long jump for track and field. Three technician specialists of the Sports Authority of Thailand, Sports Sciences Center Section 3 operated all pre and post-test tests and the same coach always administered the soccer and track and field skill tests.

Maximum strength: 1RM; the maximum load that can be lifted in one repetition $[16,17]$. All subjects were evaluated for $1 \mathrm{RM}$ in the bench press and leg press exercises by using Strive resistance training machine (Strive Enterprises, Inc., USA). The target load was obtained within 6 trials. The 1RM load was recorded in kilograms. Both exercises were performed using the same resistance equipment. 
Table 1. Complex and Resistance Training Programs

\begin{tabular}{|c|c|c|c|c|c|c|}
\hline Week & Session & Resistance Exercises & Set $X$ repetitions & Intensity (\%1RM) & Plyometric Exercises & Set $\mathrm{X}$ repetitions \\
\hline \multirow{6}{*}{1} & \multirow{3}{*}{1} & Leg press & \multirow{3}{*}{$2 \times 12$} & \multirow{3}{*}{70} & Rim jump & \multirow{3}{*}{$2 \times 10$} \\
\hline & & Bench press & & & Medicine ball chest pass & \\
\hline & & Knee extension & & & Tuck jump & \\
\hline & \multirow{3}{*}{2} & Arm curl & \multirow{3}{*}{$2 \times 12$} & \multirow{3}{*}{70} & Medicine ball overhead throw & \multirow{3}{*}{$2 \times 10$} \\
\hline & & Knee curl & & & Tuck jump & \\
\hline & & Lat pull down & & & Medicine ball backward throw & \\
\hline \multirow{6}{*}{2} & \multirow{3}{*}{3} & Leg press & \multirow{3}{*}{$3 \times 12$} & \multirow{3}{*}{70} & Rim jump & \multirow{3}{*}{$3 \times 10$} \\
\hline & & Bench press & & & Medicine ball chest pass & \\
\hline & & Knee extension & & & Tuck jump & \\
\hline & \multirow{3}{*}{4} & Arm curl & \multirow{3}{*}{$3 \times 12$} & \multirow{3}{*}{70} & Medicine ball overhead throw & \multirow{3}{*}{$3 \times 10$} \\
\hline & & Knee curl & & & Tuck jump & \\
\hline & & Lat pull down & & & Medicine ball backward throw & \\
\hline
\end{tabular}

Wingate anaerobic test: A modified set devised for the Wingate anaerobic test (Monark Ergomedic 828E, AB, Sweden) of the Sports Authority of Thailand was used to evaluate anaerobic peak power and anaerobic capacity throughout the experiments. Peak anaerobic power represents the highest power output (watts $/ \mathrm{kg}$ ) generated during first 5 seconds of test and anaerobic capacity is the total amount of work (watts $/ \mathrm{kg}$ ) accomplished over a 30 seconds. The set was composed of an adapter, in the middle of which its input was connected to the Ergomedic 828E Monark Exercise AB, Sweden and its output to a CPU. The CPU was equipped with an on-line Version 2.0/1993 of the Sports Authority of Thailand. The breaking force applied was $0.068 \mathrm{kp}$ per $\mathrm{kg}$ bodyweight and the force was equivalent to mechanical work of $4.02 \mathrm{~J}$ per pedal revolution per $\mathrm{kg}$ bodyweight. The values of the force and the mechanical work were the average values used for 13- to14-year-old boys and for 13- to 14-yearold girls [18]. These values were supposed to be used for boys and girls in Thailand. Testing procedures were according to previous suggestion [19]. After a 10-minute warm-up, a subject began pedalling as fast as possible without any resistance or breaking force. Within three seconds, a previously calculated fixed breaking force was applied to the flywheel of the Ergometer and the subject continued to pedal "all out" for 30 seconds. The testing devices were run by the technician specialist of the Sports Authority of Thailand, Sports Sciences Center Section 3.

40 yard sprint time: The 40 yard sprint test [20]was used to measure the 40 yard sprint time of subjects by using the Fusion Sport Smart Speed (Fusion Sport, Pty, Ltd, Australia). The device was operated by the technician specialist of the Sports Authority of Thailand, Sports Sciences Center Section 3. All subjects were instructed to sprint on the running track at maximum speed for a distance of 40 yards. Fusion Sport Smart Speed recording devices were placed at the start and finish points to record the time. Subjects performed two sprints, resting for five minutes between trials.

Agility (T-test): The agility of all subjects was evaluated using a T-test [21] on an outdoor court. The Fusion Sport Smart Speed (Fusion Sport, Pty, Ltd, Australia) was used to record the time of each performance. The devices were placed at the start and finish points to record the time. Sub- jects started behind the recording device and ran as fast as possible, following the $\mathrm{T}$ figure and passing the finish point. After one practice trial, two agility tests were performed. The Fusion Sport Smart Speed device was operated by the technician specialist of the Sports Authority of Thailand, Sports Sciences Center Section 3.

Vertical jump: Vertical jump performance was measured for two types of jumps, a countermovement jump (CMJ) and a squat jump (SJ) [22]. The Fusion Sport Smart Jump (Fusion Sport, Pty, Ltd, Australia) was used to record the height of each jump. Jumps were performed on the rubber plate of the Fusion device. Subjects performed each jump twice. The Fusion Sport Smart Jump was operated by the technician specialist of the Sports Authority of Thailand, Sports Sciences Center Section 3.

Soccer skills test: Two types of soccer skill tests were used: shooting and passing. These soccer skill tests are normallyused at the school to evaluate student skill.

Passing skill test: Subjects stood with a ball behind a line ten feet from a wall. They passed the ball to hit the wall, and continued passing whenever the ball returned to them. Subjects were told to pass the ball as fast as possible for $30 \mathrm{sec}-$ onds. A score was registered only when the ball was kicked and hit the wall from behind the 10-foot-distance line. Subject performed the test twice, and the best score was recorded.

Shooting skill test: Subjects shot 10 balls toward the goal without a goalkeeper from the 18 yard range. Ten balls were placed one foot apart along the 18 yard line. The goal space was divided into 6 sections, with each section giving a different score: Three points if the subject shot a ball into the upper right or left sections, two points for the upper middle or lower right or left and one point for the lower middle. A score was not counted if the subject shot a ball out of the target area. Subjects performed the test twice, and the best score was recorded.

Track and field tests: Two types of track and field tests were used: the standing long jump and the medicine ball throw. These track and field skill tests are used at the school to evaluate student skill. 
Table 2. Mean \pm Standard Deviation, mean Differences $(\Delta)$ between Pre- and Posttest, $t$ and $p$ Values from t-tests of all Variables in the Complex Training and Resistance Training Groups

\begin{tabular}{|c|c|c|c|c|c|}
\hline Complex Training Group & Pre & Post & $\Delta$ & $\mathbf{T}$ & $\mathbf{p}$ \\
\hline IRM leg press $(\mathrm{kg})$ & $144.25 \pm 26.79$ & $145.11 \pm 26.06$ & -0.86 & -2.65 & 0.08 \\
\hline Peak anaerobic power (watts $/ \mathrm{kg}$ ) & $9.34 \pm 1.61$ & $9.53 \pm 1.40$ & -0.19 & -1.36 & 0.21 \\
\hline Anaerobic capacity (watts/kg) & $7.38 \pm 1.10$ & $7.39 \pm 1.02$ & -0.02 & -0.21 & 0.84 \\
\hline Countermovement jump $(\mathrm{cm})$ & $34.50 \pm 6.38$ & $34.24 \pm 6.29$ & 0.26 & 1.38 & 0.21 \\
\hline Squat jump (cm) & $32.68 \pm 6.20$ & $32.37 \pm 6.05$ & 0.31 & 1.29 & 0.24 \\
\hline Shooting skill (pts) & $14.25 \pm 1.26$ & $14.00 \pm 1.15$ & 0.25 & 0.52 & 0.64 \\
\hline Passing skill (pts) & $14.75 \pm 2.36$ & $14.25 \pm 1.50$ & 0.5 & 1 & 0.39 \\
\hline Resistance Training Group & Pre & Post & $\Delta$ & $\mathbf{T}$ & $\mathbf{p}$ \\
\hline $1 \mathrm{RM}$ bench press $(\mathrm{kg})$ & $51.83 \pm 7.78$ & $52.69 \pm 7.42$ & -0.86 & -2.65 & 0.08 \\
\hline 1RM leg press $(\mathrm{kg})$ & $142.56 \pm 8.17$ & $142.58 \pm 7.70$ & -0.01 & -0.02 & 0.99 \\
\hline Peak anaerobic power (watts $/ \mathrm{kg}$ ) & $9.95 \pm 2.02$ & $9.65 \pm 1.93$ & 0.3 & 2.05 & 0.08 \\
\hline Anaerobic capacity (watts/kg) & $7.86 \pm 1.24$ & $7.86 \pm 1.12$ & 0 & 0.01 & 0.99 \\
\hline 40 yard speed (sec) & $5.27 \pm 0.59$ & $5.29 \pm 0.56$ & -0.02 & -1.76 & 0.12 \\
\hline Agility (sec) & $9.71 \pm 0.50$ & $9.72 \pm 0.50$ & -0.02 & -0.78 & 0.46 \\
\hline Countermovement jump $(\mathrm{cm})$ & $31.31 \pm 9.12$ & $30.77 \pm 8.93$ & 0.54 & 1.96 & 0.09 \\
\hline
\end{tabular}

Standing long jump: Subjects stood behind a line, facing the sandbox, with feet slightly apart. They jumped forward using a two foot take-off, landing with a swinging of the arms, and bending of the knees. Subjects attempted to jump as far as possible and land on both feet without falling backwards. The standing long jump distance was measured in meters. Subjects performed the test twice and the best score was recorded.

Medicine ball throw: Subjects stood at a line with the feet side by side and slightly apart, facing the sandbox toward which the ball was to be thrown. The three-kilogram medicine ball was held with the dominant hand at the shoulder then the subject threw the ball vigorously forward as far as possible. The subject was not permitted to step over the line after the ball was released. The distance from the starting line to the dropped point of the medicine ball in the sandbox was measured in meters. Subjects performed the test twice, and the best score was recorded.

\section{Data Analysis}

Data analysis consisted of several parts: (1) main descriptive statistics to show group behavior in all tests; (2) graphs with different data configurations at pre- and posttest; (3) an intraclass correlation coefficient (ICC) to assess data reliability; (4) repeatability using the Bland-Altman technique [23]. Since we will have 24 Bland-Altman plots we will only present 6 for illustrative purposes. For example, in the Wingate anaerobic test, it was expected that the coefficient of repeatability of peak anaerobic power would not be more than 1 watts $/ \mathrm{kg}$. Mean differences $(\Delta)$ were also computed to show the magnitude of changes from pre- to posttest for each variable. In addition, dependent $\mathrm{t}$-tests were used to determine whether therewere significant differences between pre- and posttests in all athletic tests. All analyses were done in SPSS 18.0.

\section{RESULTS}

Table 2 shows important statistical results that are of great help in understanding the relevance of the pilot study. Mean ( \pm standard deviation) values of the pre- and posttests of the complex and resistance training groups are reported. Mean differences $(\Delta)$ are included, indicating the amount of change from pre- to posttest. Also, $t$ and $p$ values from the dependent t-test are given. 

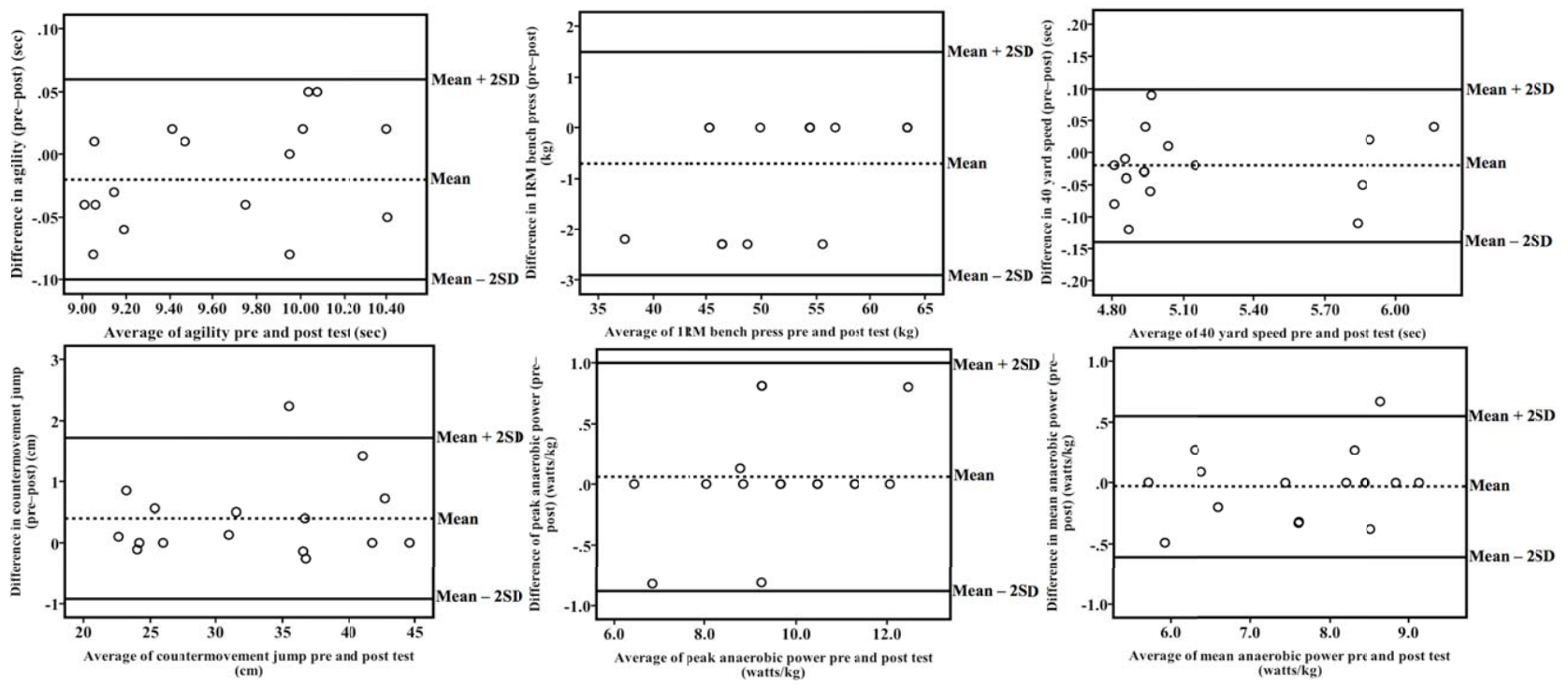

Fig. (1). Examples of six Bland-Altman plots of both complex and resistance training group (1RM bench press, countermovement jump, 40 yard speed, agility, peak anaerobic power and mean anaerobic power): Mean (dashed line) is average of difference in measured variable (preposttest). Mean - 2SD and Mean + 2SD (dark lines) are most of the difference from Mean which derived from the average of differences in measured variable $(\Delta) \pm 2 \times$ Standard deviation of the Mean difference. For example in peak anaerobic power, mean $=0.06 \mathrm{watts} / \mathrm{kg}$ and SD $=0.47$ watts $/ \mathrm{kg}$. Mean $-2 \mathrm{SD}=-0.88$ watts $/ \mathrm{kg}$ and Mean $+2 \mathrm{SD}=1$ watts $/ \mathrm{kg}$.

Mean and standard deviations of all variables are similar from pre- to posttest in both the complex training and the resistance training group. Mean differences $(\Delta)$ are very small, showing evidence of a high interindividual consistency of performance. The dependent t-test showed no statistically significant differences $(\mathrm{p}>0.05)$ in all variables. The range of ICC values of all measures was (0.67 to 0.99 ).

Fig. (1) shows only 6 of the 24 possible Bland-Altman graphs for repeatability indicating the high precision of subjects performance. For example, in the peak anaerobic performance mean differences from both complex and resistance training groups are close to $0(0.06$ watts $/ \mathrm{kg})$ with a precision interval that goes from 1 and -0.88 watts $/ \mathrm{kg}$ ); in $1 \mathrm{RM}$ bench press, mean differences are $1.1 \mathrm{~kg}$, and the interval goes from -2.91 to $1.49 \mathrm{~kg}$. For the rest of the variables, mean differences are $0.56 \mathrm{sec},-0.02 \mathrm{sec}, 0.40 \mathrm{~cm}$ and 0.03 watts $/ \mathrm{kg}$ for 40 yard speed, agility, countermovement jump and mean anaerobic power, respectively.

\section{DISCUSSION}

The current study applied a two-week pilot strength training program and used suitable testing protocols to evaluate the program's feasibility for young Thai male athletes. Subjects' responses to different strength training protocols and varied testing procedures targeted to measure muscular strength and power, physical performance and sport skills were observed. Different approaches to estimate data reliability were presented. To our knowledge, this is the first study on young Thai male athletes.

The training programs for this study were based on available data sources $[4,11,13,24,25]$ that used both children and adolescents who were either non-athletes or athletes. As our resistance training programs were predominantly designed to evaluate how young Thai male athletes respond to the program, subjective information revealed that young
Thai male athletes were highly capable of performing all exercise routines throughout the two weeks of study. During the study, all resistance exercises in each session were well tolerated by subjects. All of subjects were closely supervised by researcher and after each set of exercise, comments and suggestions were given to subjects in order to perform a proper resistance exercises technique. They were also instructed to perform in each exercise at moderate repetition velocity, which is suggested for youth resistance training program by the National Strength and Conditioning Association [2]. Performance discomfort or injuries were not observed in all resistance training sessions. No subjects dropped out during the resistance training.

The same order of exercises in the resistance training group such as training intensity, volume, and repetition velocity were applied to complex training group in order to perform exercise during resistance exercise phase. We allowed them 4 minutes rest between resistance exercise and plyometric exercise in each set as well as between exercise set. As suggested by previous study [26], this length of rest period may be optimum to enhance subsequent plyometric exercises. As plyometric exercises are characterized by stretch-shortening cycle that start with a rapid stretch of muscle (eccentric phase) and followed by a rapid shortening of the same muscle (concentric phase) [27], subjects were motivated and instructed to perform each repetition rapidly. We also focused on their quality of movements as well as instructed them to perform every repetition with proper form [14]. Comments and suggestions were given to subjects throughout every plyometric exercise phase and we also observed their improvement in performing exercises. Most of subjects were capable of performing plyometric exercises. Moreover, they were enthusiastic about participating in the training program as they come to training early and intended to perform exercises in all sessions. Additionally, according to the subjective information from coaches, subjects and 
researchers, no any discomfort in performing all exercises were observed. Moreover, no occurrence of injury as well as subject drop-out were observed during complex training program. Based on our findings and observations, it can be suggested that complex training program is handled well by young Thai male athletes and program seems to be useful for coaches to apply this training protocol to be one part of their sport training program.

Another aim of this study was to assess how reliable data can be obtained from various tests by available testing devices. As our training programs were designed for 2 weeks, we did not expect any changes in all variables because of insufficient training duration for improving strength and performances. As we expected, descriptive statistics (mean \pm SD) reported in Table 2 shows similar magnitude in all variables of both complex and resistance training groups from pre- to posttests. Moreover, mean differences $(\Delta)$ are of small magnitude (lower than 1) in all variables of both groups. Therefore, dependent t-tests reported no significantly differences between pre- and posttests in all performances of both groups $(p>0.05)$. These results clearly identified no changes from neither resistance nor complex training programs on all subject's measured performances after twoweeks training programs. High reliabilities were observed from all tests in both training groups. Results reported that intraclass correlation coefficient (ICC) in most of the tests were close to $1.00(0.82-0.99)$. Only shooting skill test in resistance training group showed low reliability $(0.67)$. These results could be interpreted that subjects showed high consistency in performances and also testing protocols and devices were reliable. Lastly, repeatability using BlandAltman plots showed good repeatabilities between pre- and posttest in all variables. As the Bland and Altman plot is a technique for examining precision between two measurements, it is expected that $95 \%$ of differences should be less than two standard deviations (this definition of a repeatability coefficient is adopted, for example, by the British Standards Institution) [28]. It is also expected that the mean differences should close to zero since the same method was used [23]. As shown in Fig. (1), precise measurements were observed in peak anaerobic power, 1RM bench press, 40 yard speed, agility, countermovement jump and mean anaerobic power for both complex and resistance training group. Furthermore, the mean differences between pre- and posttest training were close to $0(-0.06$ watts $/ \mathrm{kg}$ for anaerobic power, $1.71 \mathrm{~kg}$ for $1 \mathrm{RM}$ bench press, $-0.02 \mathrm{sec}$ for 40 yard speed, $0.40 \mathrm{~cm}$ for countermovement jump and -0.03 watts $/ \mathrm{kg}$ for mean anaerobic power). These results clearly indicate a high consistency of subject's performance as well as accurate measurements.

Although subjects in our study had no experience in all tests, we found that all tests were well tolerated by all subjects. We allowed subjects 10 minutes warm up prior to the testing session. During all testing sessions, subjects were asked by researcher and coaches to perform their best. Some tests which are considered challenging task for subjects such as $1 \mathrm{RM}$ test and the Wingate anaerobic test were found to be feasible for young male Thai athletes. The Wingate anaerobic test has also been used to measure anaerobic power from effect of complex training program on early pubescent boys [11]. Likewise, 1RM protocol was also used to measure maximum strength in adolescent soccer players before and after 16 weeks of resistance training program [4]. In recent study, we observed no injuries occurred and no subjects dropped out during all testing sessions. Additionally, we also observed no feeling of discomfort from subjects during testing.

\section{CONCLUSIONS}

This study showed that closely supervised pilot complex and resistance training programs with a very short duration are well tolerated by young athletes. Physical performance measurements operated by qualified staff provide high quality data. Young athletes showed high consistency performance in all testing procedures. These pilot training programs were feasible and harmless.

\section{LIMITATIONS AND PRACTICAL APPLICATIONS}

The major limitation of this study, considering its purposes, is related to sample diversity, meaning that greater generalizability would be achieved if we sampled athletes from other sports. Nevertheless, its practical applications are of importance. Firstly, the design of this pilot study can be applied as a primary template in other schools when planning strength training programs. Secondly, it shows a number of steps aiming at collecting high quality data to monitor strength and performance changes that are to be highly useful for coaches and athletes.

\section{CONFLICT OF INTEREST}

No funding supported this research and the author has no conflicts of interest.

\section{ACKNOWLEDGEMENTS}

We thank Khon Kaen Sport School, Thailand, and all of the young soccer players and track and field athletes who participated in the study, as well as the soccer and track and field coaches, for their kind cooperation. The authors also thank the technician specialists of the Sports Authority of Thailand, Sports Sciences Center Section 3, for their testing device services.

\section{REFERENCES}

[1] Malina RM. Weight training in youth-growth, maturation, and safety: an evidence-based review. Clin J Sports Med 2006; 16: 478-87.

[2] Faigenbaum AD, Kraemer WJ, Blimkie CJR, et al. Youth resistance training: updated position statement paper from the National Strength and Conditioning Association. J Strength Cond Res 2009; 23: S60-79.

[3] McCambridge TM, Stricker PR. Strength training by children and adolescents. Pediatrics 2008; 121:835-40.

[4] Christou M, Smilios I, Sotiropoulos K, Volaklis K, Pilianidis T, Tokmakidis SP. Effects of resistance training on the physical capacities of adolescent soccer players. J Strength Cond Res 2006; 20: 783-91.

[5] Szymanski DJ, Szymanski JM, Bradford TJ, Schade RL, Pascoe DD. Effect of twelve weeks of medicine ball training on high school baseball players. J Strength Cond Res 2007; 21: 894-901.

[6] Wong PL, Chamari K, Wisloff U. Effects of 12-week on-field combined strength and power training on physical performance among U-14 young soccer players. J Strength Cond Res 2010; 24: 644-52.

[7] Diallo O, Dore E, Duche P, Van Praagh E. Effects of plyometric training followed by a reduced training programme on physical 
performance in prepubescent soccer players. J Sports Med Phys Fitness 2001; 41: 342-8.

[8] Meylan C, Malatesta D. Effects of in-season plyometric training within soccer practice on explosive actions of young players. J Strength Cond Res 2009; 23: 2605-13.

[9] Docherty D, Robbins D, Hodgson M. Complex training revisited: a review of its current status as a viable training approach. Strength Cond J 2004; 26: 52-7.

[10] Ebben WP, Watts PB. A review of combined weight training and plyometric training modes: complex training. Strength Cond J 1998; 20: 18-27.

[11] Ingle L, Sleap M, Tolfrey K. The effect of a complex training and detraining programme on selected strength and power variables in early pubertal boys. J Sports Sci 2006; 24: 987-97.

[12] Maio Alves JM, Rebelo AN, Abrantes C, Sampaio J. Short-term effects of complex and contrast training in soccer players' vertical jump, sprint, and agility abilities. J Strength Cond Res 2010; 24 : 936-41.

[13] Santos EJAM, Janeira MAAS. Effects of complex training on explosive strength in adolescent male basketball players. J Strength Cond Res 2008; 22: 903-9.

[14] Chu DA, Faigenbaum AD, Falkel JE. Progressive Plyometric for Kids. Monterey, CA: Healthy Learning 2006.

[15] Kraemer WJ, Fleck SJ. Strength Training for Young Athletes. $2^{\text {nd }}$ ed. Champaign, IL: Human Kinetics 2005.

[16] Faigenbaum AD, Milliken LA, Westcott WL. Maximal strength testing in healthy children. J Strength Cond Res 2003; 17: 162-6.

[17] Ramsay JA, Blimkie CJ, Smith K, Garner S, MacDougall JD, Sale DG. Strength training effects in prepubescent boys. Med Sci Sports Exerc 1990; 22: 605-14.

[18] Dotan R, Bar-Or O. Load optimization for the Wingate Anaerobic Test. Eur J Appl Physiol Occup Phys 1983; 51: 409-17.
[19] Bar-Or O. The Wingate anaerobic test. An update on methodology, reliability and validity. Sports Med 1987; 4: 381-94.

[20] Cook G. Baseline sport-fitness testing. In: Foran B, Ed. HighPerformance Sports Conditioning. Champaign, IL: Human Kinetics 2001.

[21] Semenick D. Tests and measurements: the T test. Natl Strength Cond Assoc J 1990; 12: 36-7.

[22] Bosco C, Luhtanen P, Komi PV. A simple method for measurement of mechanical power in jumping. Eur J Appl Physiol Occup Phys 1983; 50: 273-82.

[23] Bland JM, Altman DG. Statistical methods for assessing agreement between two methods of clinical measurement. Lancet 1986; 1: 307-10.

[24] Faigenbaum AD, McFarland JE, Keiper FB, et al. Effects of a short-term plyometric and resistance training program on fitness performance in boys age 12 to 15 years. J Sports Sci Med 2007; 6: $519-25$

[25] Lillegard WA, Brown EW, Wilson DJ, Henderson R, Lewis E Efficacy of strength training in prepubescent to early postpubescent males and females: effects of gender and maturity. Pediatr Rehabil 1997; 1: 147-57.

[26] Jensen RL, Ebben WP. Kinetic analysis of complex training rest interval effect on vertical jump performance. J Strength Cond Res 2003; 17: 345-9.

[27] Bosco C, Komi PV. Potentiation of the mechanical-behavior of the human skeletal-muscle through prestretching.Acta Physiol Scand 1979; 106: 467-72.

[28] Institution BS. Precision of Test Methods 1: Guide for the determination and reproducibility for a standard test method. London: BSI 1975.

(C) Buranarugsa et al.; Licensee Bentham Open.

This is an open access article licensed under the terms of the Creative Commons Attribution Non-Commercial License (http://creativecommons.org/licenses/ by-nc/3.0/) which permits unrestricted, non-commercial use, distribution and reproduction in any medium, provided the work is properly cited. 